\title{
The Truncated Icosahedron as an Inflatable Ball
}

\author{
Tibor Tarnai', András Lengyel ${ }^{1 *}$ \\ 1 Department of Structural Mechanics, Faculty of Civil Engineering, Budapest University of Technology and Economics, \\ H-1521 Budapest, P.O.B. 91, Hungary \\ * Corresponding author, e-mail: lengyel.andras@epito.bme.hu
}

Received: 09 April 2018, Accepted: 20 June 2018, Published online: 29 October 2018

\begin{abstract}
In the late 1930s, an inflatable truncated icosahedral beach-ball was made such that its hexagonal faces were coloured with five different colours. This ball was an unnoticed invention. It appeared more than twenty years earlier than the first truncated icosahedral soccer ball. In connection with the colouring of this beach-ball, the present paper investigates the following problem: How many colourings of the dodecahedron with five colours exist such that all vertices of each face are coloured differently? The paper shows that four ways of colouring exist and refers to other colouring problems, pointing out a defect in the colouring of the original beach-ball.
\end{abstract}

\section{Keywords}

polyhedron, truncated icosahedron, compound of five tetrahedra, colouring of polyhedra, permutation, inflatable ball

\section{Introduction}

Spherical forms play an important role in different fields of science and technology, and in different areas of everyday life. For example, spherical domes are quite common in architecture, and spherical balls are used in most ball games. Spherical inflatables such as air-supported radomes (radar or radio antenna domes) and soccer balls have structures analogous to each other. They are made from planar elements that are stitched (glued) together, and after inflation, they achieve a shape approximating a sphere. The present paper is related to inflatable balls.

These multi-panel balls, which originally were stuffed with hair or feathers, have a long history, although their beginnings are not known to us. We know that Plato (1892a) wrote in Phaedo (360BC): "... the earth, when looked at from above, is in appearance streaked like one of those balls, which have leather coverings in twelve pieces, and is decked with various colours ...". Referring to Timaeus (Plato, 1892b), Robin (1935) remarked in a footnote that the number of faces of a regular dodecahedron is twelve. Rassat and Thuillier (1996) went further, and explicitly stated that a dodecahedral leather ball is mentioned in the quotation above. We prefer to think that this ball could be dodecahedral, but not necessarily. On the one hand, the emphasised number twelve could refer to many other things, and on the other, we do not know of any archaeological evidence for the existence of dodecahedral balls
- not even among the relics of the Romans who inherited many ball games from the Greeks.

The Romans mainly used balls composed of equal digonal panels, forming a regular hosohedron (Coxeter, 1973, p. 12; Wikipedia (a)). These balls were colourful, as seen in the $4^{\text {th }}$ century AD mosaic in the Villa Romana del Casale, Piazza Armerina, Sicily (Wikipedia (b)); they could be composed of as many as twelve digonal pieces as demonstrated by toy balls, found in Egypt from the Roman period, kept in the British Museum (2017).

During the Han (202BC-220AD) and Tang (618-907AD) dynasties in China, tetragonal and hexagonal hosohedral balls were used to play cuju (ancient Chinese football) (Cui, 1991). During the Tang dynasty, the hexagonal hosohedral balls were modified. The two vertices of the hosohedron were truncated. The ball obtained in this way was built from two hexagonal and six quadrilateral panels. The Chinese claim that, during the Song dynasty (9601279AD), a 12-piece ball was developed where each panel was a regular pentagon (hwjyw.com). We do not know the details of this, but it is well known that there are paintings and drawings from the Song to the Ming dynasties that show people playing cuju with a ball on which (at least) one pentagon is seen.

We know of only one example of the existence of polyhedral balls in the ancient times. This is a ball that can be 
seen in the $2^{\text {nd }}$ century AD mosaic of the Baths of Porta Marina, Ostia (Rassat and Thuillier, 1996). It could be a hexadecahedron of $D_{2}$ symmetry with 4 hexagonal and 12 pentagonal faces. Since manufacturing such a complicated ball must be difficult, Rassat and Thuillier (1996) thought that the mosaic is probably just a misinterpretation of a dodecahedron.

During the Renaissance, artists and scientists studied the Archimedean polyhedra. Pacioli (1509) published them with the drawings by Leonardo da Vinci. One of these polyhedra is the truncated icosahedron of $I_{h}$ symmetry with 12 pentagonal and 20 hexagonal faces (Fig. 1). It looks quite round, and could serve as a basis for balls, but as far as we know it was not used for this purpose, but, for instance, much later for decorating a church memorial (Fig. 2) (Tarnai and Krähling, 2008). However, ball games were also played in the Renaissance. One piece of evidence for this is a leather ball in the Stirling Smith Art Gallery and Museum, Scotland, which was made sometime in the $1540 \mathrm{~s}$, and is considered as the world's oldest extant football (The Stirling Smith Art Gallery and Museum). It was constructed from only three panels: two equal circles and an elongated rectangle. Prior to inflation it was shaped like a circular cylinder. Interestingly, this kind of ball is still in use in England in the traditional Shrovetide football game (Wikipedia (c)). With the growing popularity of football, the number of different ball designs increased, and around the turn of the $20^{\text {th }}$ century, designs showed great variety, and in addition to the hosohedral ball (Fig. 3(a)), many variants occurred (Tarnai, 2005). (The ancient hosohedron form still exists in present-day beach-balls (Fig. 3(c)).)

The truncated icosahedral ball appeared only in the 1960s. Most people think that this 32-panel ball (Fig. 3(b)) is an Adidas invention, since Adidas made the first official FIFA World Cup match ball in 1970, which was a truncated icosahedral ball. However, the 32-panel truncated icosahedral ball was, in fact, invented by Eigil Nielsen, a former goal keeper of the Danish national football team and was introduced by his firm Select in 1962 (Select Sport (a), (b)). (Adidas started making footballs only in 1963 (Wikipedia (d)).) We do not know the circumstances of the invention or how Nielsen got the idea, but it is clear that it required a certain level of geometrical knowledge. As Michael Karlsen of Select (personal communication) informed us, Eigil Nielsen knew a mathematical family. His invention has never been patented. His philosophy was that the ball is the most entertaining toy in the world,

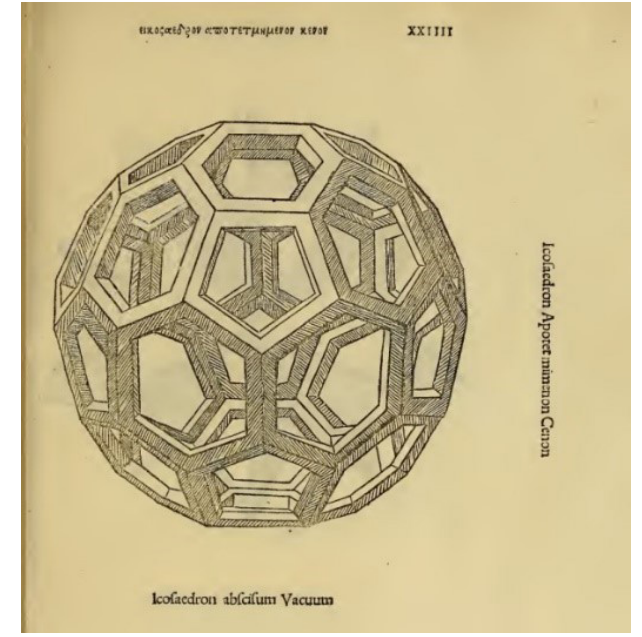

Fig. 1 A lath skeleton model of a truncated icosahedron drawn by Leonardo da Vinci (Pacioli, 1509). (Source: https://archive.org/details/ divinaproportion00paci)

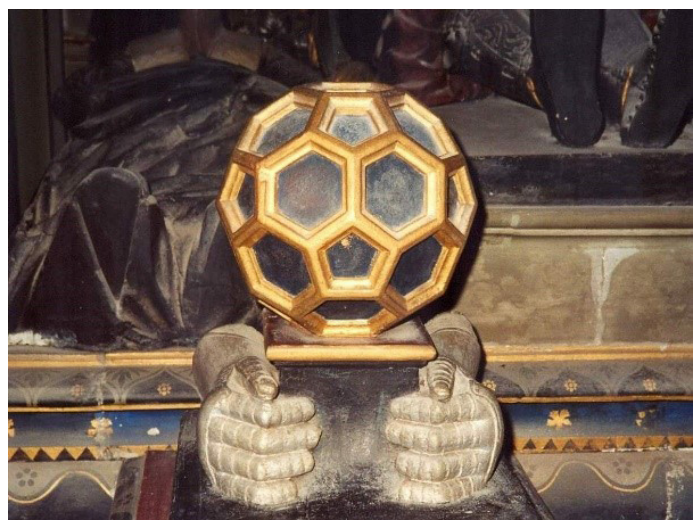

Fig. 2 A truncated icosahedron at the feet of the effigies on the monument to Sir Anthony Ashley in Wimborne St. Giles, England, erected in 1627. (Photo: Tarnai). The respected architecture historian Nikolaus Pevsner misdescribed this object as a "sphere of hexagons" (Newman and Pevsner, 1975).

and "we should make sure that every step to improve its qualities is free for all of us". As far as we know, the first patent was given in 1964 for the black and white colouring of the ball (Doss, 1964).

The date of the invention of the truncated icosahedral ball has been known to us for years. Consequently, it was a great surprise when, while watching a recent TV documentary (Kloft, 2017), one of us unexpectedly saw a colourful truncated icosahedral beach-ball (Fig. 3(d)) on the screen. This documentary showed excerpts of several archive home movies in colour. The ball appeared in the home movie entitled Wörthsee, made sometime at the end of the 1930s. This means that a truncated icosahedral ball - hereafter we will call it simply the "Wörthsee ball" - already 


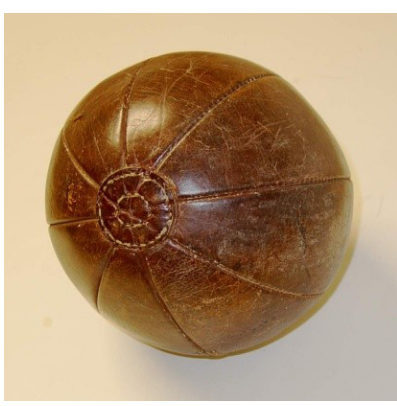

(a)

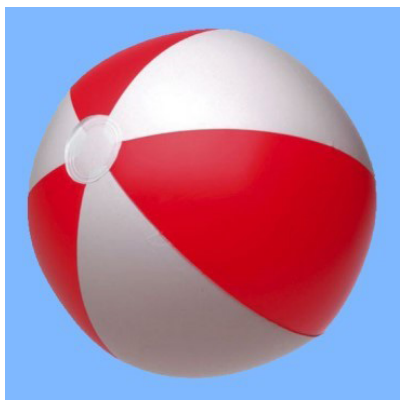

(c)

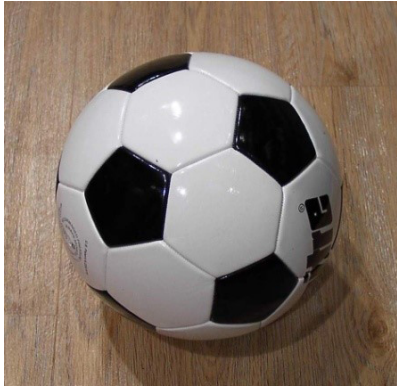

(b)

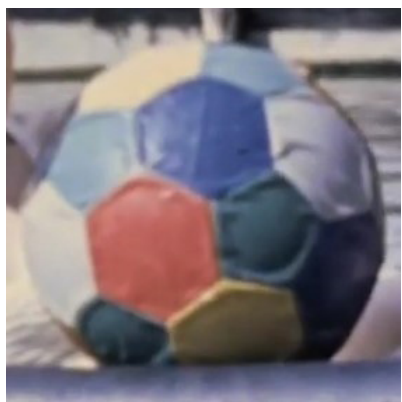

(d)
Fig. 3 Hosohedral balls composed of digonal panels (a), (c), and truncated icosahedral balls (b), (d). (a) Soccer ball in the second half of the $19^{\text {th }}$ century, from the collection of the National Football Museum, Preston, UK. (Photo: Tarnai). (b) Modern 32-panel soccer ball. (Photo: Tarnai). (c) Beach-ball. (Available at: https://www. evapresent.eu/). (d) "Wörthsee ball", a beach-ball from the end of the 1930s. (Kloft, 2017).

existed more than twenty years before Eigil Nielsen's 32-panel ball, although this was not common knowledge.

The aim of the present paper is to describe the properties of the Wörthsee ball, to point out a defect in its colouring and show how to "correct" the colouring, and finally, to provide data for making conjectures about the origin of this ball.

\section{The Wörthsee ball}

The date of filming of the Wörthsee ball was very close to the 1936 Summer Olympics. The seam pattern of the official balls used there in football, basketball and water polo was a cube, the faces of which were cut in half. Thus, these balls had "leather coverings in twelve pieces" and $T_{h}$ symmetry. It is worth mentioning that one rectangular "piece" (panel) is combinatorially a pentagon, and therefore the shape of such a ball is a spherical pentagonal dodecahedron, but with irregular pentagons. It is considered, that from such a ball, it is not possible to develop gradually a truncated icosahedral ball. This is why the Wörthsee ball was a revolutionary new design. Despite searching for photos of beach-balls of the time, it was not possible to find another copy of the Wörthsee ball. It is reasonable to

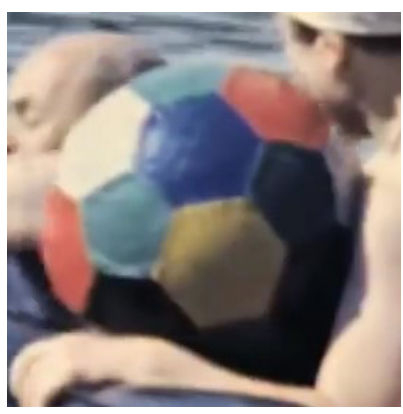

(a)

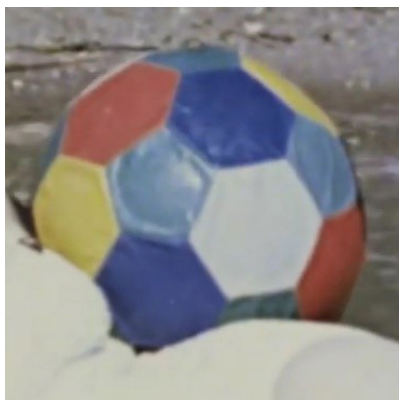

(c)

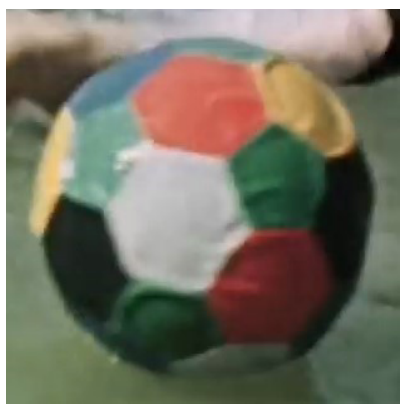

(b)

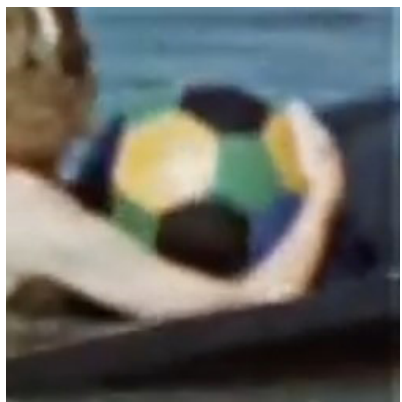

(d)
Fig. 4 Different views of the Wörthsee ball. Colours in counterclockwise order around a pentagon: (a) R, K, Y, B, W,

(b) R, B, Y, K, W and R, K, Y, R, W, (c) R, Y, B, W, B (d) B, Y, K, Y, K. (Kloft, 2017)

assume that this ball was made as a one-off, and not by mass production. It seems that beach-balls, similar to those of the present day, were mostly hosohedra at that time.

The skin of the Wörthsee ball was made of thin, coloured textile pentagons and hexagons. The seams were quite robust. Figs. 3(d) and 4 show, that in the neighbourhood of some of the seams, the Gaussian curvature is negative, and there are some wrinkles that cannot occur at an inflated spherical membrane. At the same time, the central parts of many polygons look tight, as if the wet membrane is sticking to a smooth spherical surface beneath. These observations suggest that the ball had a two-layer construction. The external skin was supported internally by an inflatable bladder that could be a commercial beachball itself. The robust seams, the two-layer construction unusual in beach-balls, and a defect in the colouring, to be discussed later, all corroborate the supposition that the Wörthsee ball was a home-made object.

The Wörthsee ball appeared in the documentary (Kloft, 2017) in many different positions (Fig. 4), and so it was possible to identify the complete colour pattern of the ball that is shown on a Schlegel diagram (Coxeter, 1961, p. 152) in Fig. 5(a). The pentagons were green. The hexagons were made with five different colours (black, blue, 


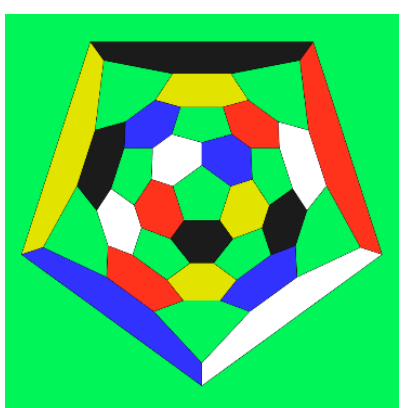

(a)

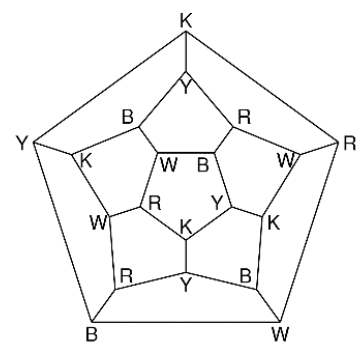

(b)
Fig. 5 Colouring the Wörthsee ball. (a) Schlegel diagram of the truncated icosahedron with coloured faces. (b) Schlegel diagram of the corresponding regular dodecahedron with coloured vertices.

red, white, yellow), and every colour was used for four hexagons. To simplify the discussion, we use the following notation for colours: $\mathrm{K}$ for black, $\mathrm{B}$ for blue, $\mathrm{R}$ for red, W for white, Y for yellow. Mathematically, colouring the hexagons of a truncated icosahedron is the same as colouring the faces of an icosahedron, or colouring the vertices of a dodecahedron. For analysis we will use the last version. Accordingly, the colouring scheme of the hexagons of the Wörthsee ball is shown on a dodecahedron in Fig. 5(b).

The edges and vertices of a dodecahedron form a graph. The distance between two vertices of the dodecahedron is defined according to graph theory. A path is a finite sequence of edges connecting a sequence of distinct vertices. The length of a path is the number of edges in the path. The distance between two vertices is the length of the shortest path joining these two vertices.

When colouring the vertices of the dodecahedron with five colours, each for four vertices, we require that it should be a valid colouring, that is, no two vertices, between which the distance is one, share the same colour. In addition, we require that the colours should be distributed uniformly, which means that the least distance between any two of the vertices coloured alike should be as large as possible, and the minimum number of different colours occurring at the vertices of a pentagon should be a maximum. In a simple formulation, the task is to colour the vertices of a dodecahedron with five colours so that all vertices of each face are coloured differently.

It seems that the designer of the Wörthsee ball had a mathematical approach, and tried to follow these requirements on the truncated icosahedron. The findings are described in terms of colouring the vertices of the corresponding regular dodecahedron.
Consider Fig. 5(b), which shows the Schlegel diagram of the dodecahedron, the vertices of which are coloured according to the hexagons of the Wörthsee ball. It is easy to see that the antipodal vertices have the same colour. Such a colouring will be called antipodal colouring. Antipodality provides the largest distance (i.e. five) between two vertices. Each vertex of the central pentagon has a different colour, and of course this is also valid for the antipodal pentagon. There is a pentagon adjacent to the central pentagon (together with its antipodal counterpart) such that its vertices have different colours. In Fig. 5(b), this pentagon has the edge BY in common with the central pentagon. It turns out that there are no other pentagons where all five vertices are coloured differently; further, there is a pentagon (together with its antipodal counterpart) with only three colours. (On the ball (Fig. 4(d)), there is a pentagon around which black and yellow hexagons occur twice.) In Fig. 5(b), this pentagon has the edge KY in common with the central pentagon. The dodecahedron representation of the Wörthsee ball has four pentagons with five colours, six pentagons with four colours, and two pentagons with three colours. Additionally, the distances between vertices of the same colour are two, three and five. Thus, we can establish that the number of different colours is not equal to five in all pentagons, and the minimum (i.e. two) and maximum (i.e. five) distances between vertices coloured alike significantly differ from each other. Therefore, the Wörthsee ball is not coloured uniformly, i.e. the colouring is erroneous in this sense.

The next task was to investigate whether it would be possible to improve the colouring of the Wörthsee ball if the property that the antipodal hexagons are coloured alike is maintained. For this purpose, consider the corresponding dodecahedron and take a cube inscribed in the dodecahedron (Fig. 6(a)). Then consider two space diagonals of the cube that span a rectangle (shaded in Fig. 6(a)), which is shown also in Fig. 6(b). Consider five copies of this rectangle obtained by its rotation about the vertical axis of the dodecahedron by angles $2 \pi k / 5, k=0,1,2,3,4$, that together form a nolid of $S_{10}$ symmetry composed of five rectangles. The vertices of a rectangle are coloured alike, and to each rectangle a different colour is assigned. This colouring (Fig. 6(b)) provides two pentagons with five colours and ten pentagons with four colours, and the distances between vertices of the same colour are two, three and five. This colouring is better than that of the Wörthsee ball, because there are no pentagons with three colours in it. (This is not the only antipodal colouring of 


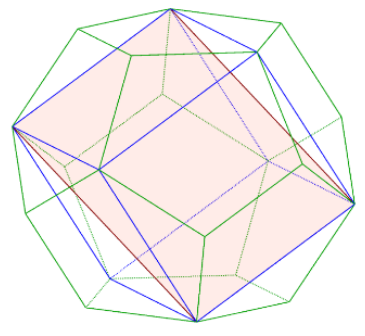

(a)

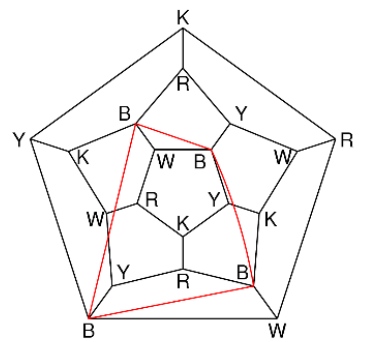

(b)
Fig. 6 (a) A rectangle inscribed in the regular dodecahedron, and (b) the corresponding colouring.

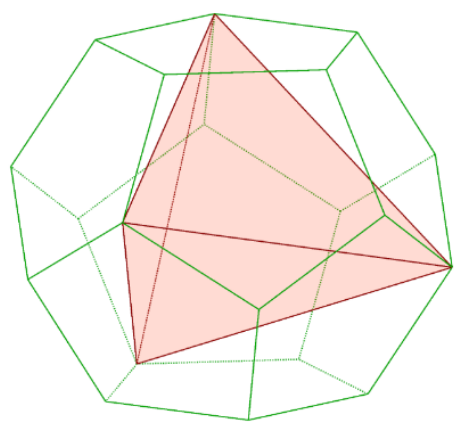

Fig. 7 A regular tetrahedron inscribed in the regular dodecahedron

the vertices of a dodecahedron with five colours, which provides two pentagons with five colours and ten pentagons with four colours, although, the other colourings will not be discussed here). In this manner, however, we are unable to improve the colouring any further.

To produce a better colouring, we have to drop the property that four vertices of the same colour form two antipodal pairs and have to select four vertices of the dodecahedron that maximize the smallest distance between pairs of these vertices. A similar problem is known in discrete geometry: How must four points be distributed on the surface of the unit sphere so as to maximize the minimum spherical distance between any two of them? The solution to this problem is known, and in the optimum arrangement the four points are the vertices of a regular tetrahedron (Fejes Tóth, 1972). The vertices of the tetrahedron fit to the vertices of the dodecahedron, that is, it can be inscribed in the dodecahedron (Fig. 7). Thus, the regular tetrahedron also maximizes the minimum distance between any two of four vertices of the regular dodecahedron.

\section{The correct colouring}

Cundy and Rollett (1981) showed an example of colouring the icosahedron with five colours such that the five faces at every vertex are coloured differently, but neither explained how that colouring was obtained, nor said how many ways this can be done. This raises the following, which is the dual formulation of the previous question: How many colourings of the dodecahedron with five colours exist such that all vertices of each face are coloured differently?

Before giving the answer let us investigate how the four vertices having the same colour should be arranged on the dodecahedron. Consider the Schlegel diagram of the dodecahedron. Let one of the vertices (a small encircled dot in Fig. 8) be coloured, say, blue. The first-neighbour vertex cannot be coloured blue (Fig. 8(a)), because we want to achieve a valid colouring, where occurrence of two adjacent vertices with the same colour is not allowed. The second-neighbour vertex (which can be selected in two different ways: left-handed and right-handed) (Fig. 8(b)) cannot be coloured blue either, because in this case there would be a pentagon, two vertices of which would have the same colour, in contradiction to the requirement that every vertex of a pentagon should be coloured differently. Following the left-handed version, we can again select two different third-neighbour vertices. The left vertex is actually a second neighbour if we approach it along another path, thus it

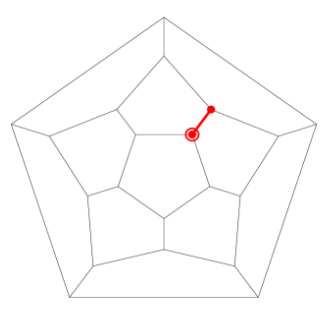

(a)

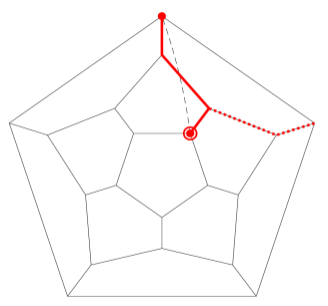

(c)

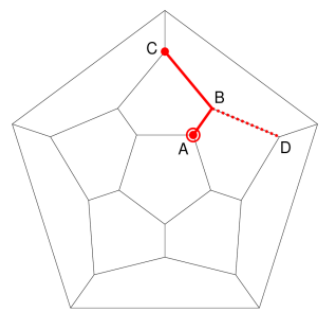

(b)

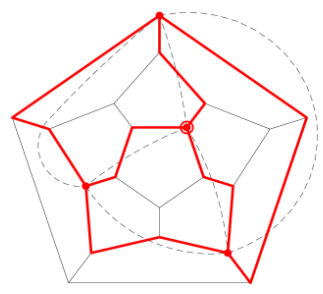

(d)
Fig. 8 Minimum distance between the identically coloured vertices (solid lines define left-handed, dashed lines define right-handed versions). (a) Not allowed - two identical colours at two pentagons, (b) not allowed - two identical colours at one pentagon.

(c) Correct, can be repeated in two additional directions, which results in a tetrahedron $(\mathrm{d})$. 
cannot be coloured blue. The right one, however, is proper and can be blue (Fig. 8(c)). Therefore, the third-neighbour vertex determines the shortest distance from one vertex to another having the same colour. Since the starting vertex is a three-valent vertex, there are two additional vertices coloured blue in the remaining two directions if the path of length three (solid line in Fig. 8(c)) is rotated about the starting vertex by angles $2 \pi k / 3, k=1,2$. The three vertices that are at distance three from the starting vertex, are also at distance three from each other (Fig. 8(d)), that is to say, the four vertices having the same colour are vertices of a regular tetrahedron inscribed in the dodecahedron (Fig. 7).

Therefore, the distance between two vertices of the tetrahedron is the maximum of the minimum distances between two vertices of the vertex quartets of the dodecahedron, and at the same time, the minimum of the distances between two identically coloured vertices of the dodecahedron, if all vertices of each face of the dodecahedron are coloured differently.

In this way, we obtain five tetrahedra, one for each colour. They can be arranged as the compound of five tetrahedra of I symmetry (Fig. 9), where the five tetrahedra are inscribed in a dodecahedron (Coxeter, 1973, p. 49; Wenninger, 1974). Fig. 9 shows clearly that this is a chiral object.

Endre Makai (personal communication) has shown that, in the case of the compound of five differently coloured tetrahedra, it is easy to demonstrate that the vertices of each face of the dodecahedron are coloured by different cyclic permutations of the five colours, and these cyclic permutations are either all even or all odd. In other words, in the case of even permutations, all cyclic permutations are present, and each occurs exactly once, with the same holding for the case of odd permutations. Consider the Schlegel diagram of the dodecahedron in Fig. 10(b) and suppose that we can get from one vertex to an identically coloured third-neighbour vertex only along a left-handed

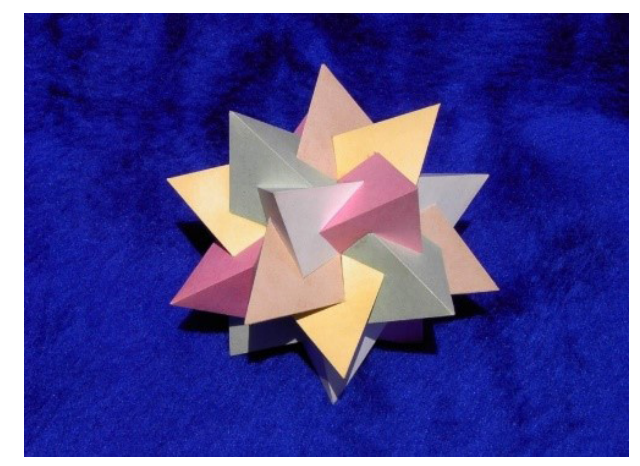

Fig. 9 Paperboard model of the compound of five tetrahedra, made by Magnus Wenninger, 1988. (Photo: Tarnai) path (solid line in Fig. 8(c)). Let the vertices of the central pentagon 1 be coloured by R, K, Y, B, W in counterclockwise. The vertex $\mathrm{R}$ has one uncoloured neighbour, which has to have a colour different from its already coloured first and second neighbours. That is, it has a colour B or Y. By applying the left-handed path to the vertices B and $\mathrm{Y}$ of pentagon 1, we find that this colour is B. Similarly, the vertex of pentagon 1 coloured by $\mathrm{K}$ has one yet uncoloured neighbour that must have a colour $\mathrm{W}$ or $\mathrm{B}$. However, it is a second neighbour of the vertex most recently coloured by $\mathrm{B}$, so it must have colour W. Similarly, moving counterclockwise results in the colours B, W, R, K, Y, in this order, at the first neighbours of vertices R, K, Y, B, W of pentagon 1, respectively. Then, all the vertices of distance two from pentagon 1 have already uniquely determined colours: Y, B, W, R, K in pentagons 2, 3, 4, 5, 6, respectively. There remains the outer pentagon 7. Its topmost vertex is an uncoloured neighbour of vertex $\mathrm{R}$ of pentagon 5 , that has a colour different from its already coloured first neighbours. So, it has a colour $\mathrm{W}$ or B. By applying the left-handed path to the vertices $\mathrm{W}$ and $\mathrm{B}$ of pentagon 5, we find that this colour must be B. Going around, similarly, the colouring in Fig. 10(b) is achieved as the only possible choice. If right-handed paths connect third-neighbour vertices of the same colour, then the enantiomorphic counterpart of this colouring pattern is obtained. (Each of two geometrical forms, which are mirror images that cannot be superposed by a continuous motion, is called enantiomorph (Coxeter, 1961, p. 276).)

Looking at Fig. 10(b), there are no two pentagons with the same cyclic permutation of the five colours, taken counterclockwise. (For the case of the outer pentagon BWRKY, counterclockwise is understood as looking at the outer pentagon from below, therefore it is clockwise when the outer pentagon is seen from above.) This is why it is worth investigating the colour permutations. All cyclic permutations of the five colours were determined, and the numbers of inversions in the permutations calculated (Wikipedia (e)); the results are presented in Table 1. To simplify the overview of the data, we used positive integers instead of capital letters to denote the colours. The five colours have 24 cyclic permutations, from which we separated the 12 even and 12 odd permutations in Tables 2 and 3, respectively. Fig. 10(b) shows that all 12 even permutations of the colours are present in the solution to the colouring problem. Hence, the 12 odd permutations should indicate something in colouring. Indeed, for example, if the white and blue colours are interchanged 


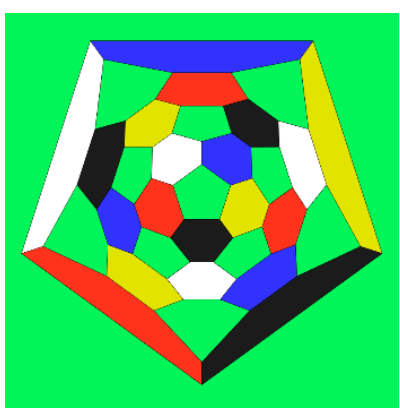

(a)

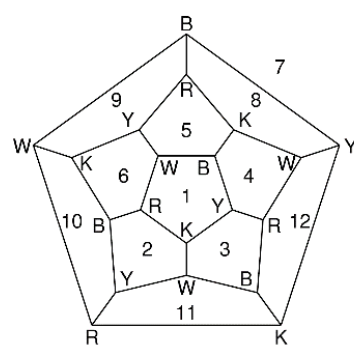

(b)
Fig. 10 First solution to correct colouring of the ball. (a), and (b) as in caption of Fig. 5.

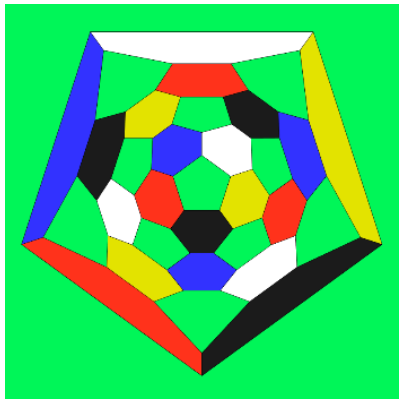

(a)

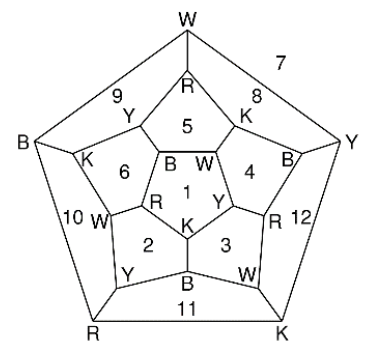

(b)
Fig. 11 Second solution to correct colouring of the ball. (a), and (b) as in caption of Fig. 5.

in Fig. 10(b), a new colouring is presented in Fig. 11(b) where all colour permutations are odd. Here, it is also possible to produce the enantiomorphic counterpart of this new colouring. As shown above, a given colour permutation of one pentagon uniquely determines the colouring of the whole dodecahedron. Since there is no other permutation in addition to the 12 even and 12 odd permutations, it follows that there exist no more colourings. In summary, we can answer the question asked at the beginning of the present Section. There exist four colourings of the dodecahedron with five colours such that all vertices of each face are coloured differently. These are: two in Figs. 10(b) and 11(b), and their two enantiomorphic counterparts. That means that the Wörthsee ball could have been coloured correctly in four different ways. Two possibilities are shown in Figs. 10(a) and 11(a).

Rouse Ball and Coxeter (1987), referring to L. B. Tuckerman, state without any proof that "the faces of an icosahedron can be coloured with five colours so that each face and its three neighbours have four different colours", and this can be done in four different ways. Endre Makai (personal communication), however, pointed out that
Table 1 Cyclic permutations of the colours

\begin{tabular}{lcc|ccc}
\hline No. & permutation $^{\text {a }}$ & $\begin{array}{l}\text { number of } \\
\text { inversions }\end{array}$ & No. & permutation $^{\text {a }}$ & $\begin{array}{c}\text { number of } \\
\text { inversions }\end{array}$ \\
\hline 1 & $(1,2,3,4,5)$ & 0 & 13 & $(1,4,2,3,5)$ & 2 \\
2 & $(1,2,3,5,4)$ & 1 & 14 & $(1,4,2,5,3)$ & 3 \\
3 & $(1,2,4,3,5)$ & 1 & 15 & $(1,4,3,2,5)$ & 3 \\
4 & $(1,2,4,5,3)$ & 2 & 16 & $(1,4,3,5,2)$ & 4 \\
5 & $(1,2,5,3,4)$ & 2 & 17 & $(1,4,5,2,3)$ & 4 \\
6 & $(1,2,5,4,3)$ & 3 & 18 & $(1,4,5,3,2)$ & 5 \\
7 & $(1,3,2,4,5)$ & 1 & 19 & $(1,5,2,3,4)$ & 3 \\
8 & $(1,3,2,5,4)$ & 2 & 20 & $(1,5,2,4,3)$ & 4 \\
9 & $(1,3,4,2,5)$ & 2 & 21 & $(1,5,3,2,4)$ & 4 \\
10 & $(1,3,4,5,2)$ & 3 & 22 & $(1,5,3,4,2)$ & 5 \\
11 & $(1,3,5,2,4)$ & 3 & 23 & $(1,5,4,2,3)$ & 5 \\
12 & $(1,3,5,4,2)$ & 4 & 24 & $(1,5,4,3,2)$ & 6 \\
\hline
\end{tabular}

${ }^{a}$ Numbers denote colours: $1=\mathrm{R}, 2=\mathrm{K}, 3=\mathrm{Y}, 4=\mathrm{B}, 5=\mathrm{W}$

Table 2 Even permutations of the colours providing the first solution

\begin{tabular}{lcc|lcc}
\hline $\begin{array}{l}\text { Penta- } \\
\text { gon }^{\mathrm{a}}\end{array}$ & $\begin{array}{c}\text { permuta- } \\
\text { tion }^{\mathrm{b}}\end{array}$ & $\begin{array}{c}\text { number of } \\
\text { inversions }\end{array}$ & $\begin{array}{l}\text { Penta- } \\
\text { gon }^{\mathrm{a}}\end{array}$ & $\begin{array}{c}\text { permuta- } \\
\text { tion }^{\mathrm{b}}\end{array}$ & $\begin{array}{c}\text { number of } \\
\text { inversions }\end{array}$ \\
\hline 1 & $(1,2,3,4,5)$ & 0 & 7 & $(1,5,4,3,2)$ & 6 \\
2 & $(1,4,3,5,2)$ & 4 & 8 & $(1,2,5,3,4)$ & 2 \\
3 & $(1,3,2,5,4)$ & 2 & 9 & $(1,4,5,2,3)$ & 4 \\
4 & $(1,5,2,4,3)$ & 4 & 10 & $(1,3,4,2,5)$ & 2 \\
5 & $(1,3,5,4,2)$ & 4 & 11 & $(1,2,4,5,3)$ & 2 \\
6 & $(1,5,3,2,4)$ & 4 & 12 & $(1,4,2,3,5)$ & 2 \\
\hline
\end{tabular}

${ }^{a}$ Numbers denote pentagons in Fig. 10(b)

${ }^{\mathrm{b}}$ Numbers denote colours: $1=\mathrm{R}, 2=\mathrm{K}, 3=\mathrm{Y}, 4=\mathrm{B}, 5=\mathrm{W}$

Table 3 Odd permutations of the colours providing the second solution

\begin{tabular}{lcc|lcc}
\hline $\begin{array}{l}\text { Penta- } \\
\text { gon }^{\text {a }}\end{array}$ & $\begin{array}{c}\text { permuta- } \\
\text { tion }^{\text {b }}\end{array}$ & $\begin{array}{c}\text { number of } \\
\text { inversions }\end{array}$ & $\begin{array}{l}\text { Penta- } \\
\text { gon }^{\text {a }}\end{array}$ & $\begin{array}{c}\text { permuta- } \\
\text { tion }^{\text {b }}\end{array}$ & $\begin{array}{c}\text { number of } \\
\text { inversions }\end{array}$ \\
\hline 1 & $(1,2,3,5,4)$ & 1 & 7 & $(1,4,5,3,2)$ & 5 \\
2 & $(1,5,3,4,2)$ & 5 & 8 & $(1,2,4,3,5)$ & 1 \\
3 & $(1,3,2,4,5)$ & 1 & 9 & $(1,5,4,2,3)$ & 5 \\
4 & $(1,4,2,5,3)$ & 3 & 10 & $(1,3,5,2,4)$ & 3 \\
5 & $(1,3,4,5,2)$ & 3 & 11 & $(1,2,5,4,3)$ & 3 \\
6 & $(1,4,3,2,5)$ & 3 & 12 & $(1,5,2,3,4)$ & 3 \\
\hline
\end{tabular}

${ }^{a}$ Numbers denote pentagons in Fig. 11(b)

${ }^{\mathrm{b}}$ Numbers denote colours: $1=\mathrm{R}, 2=\mathrm{K}, 3=\mathrm{Y}, 4=\mathrm{B}, 5=\mathrm{W}$

the condition here can be presented in the dual form in Fig. 8(b), where vertex B represents a face of the icosahedron and vertices A, C, D represent its three neighbours. According to the observations at the beginning of this Section 3, if a vertex has a colour, then none of its firstand second-neighbour vertices are allowed to have the same colour. In this case, points A, B, C, D are all first or 
second neighbours of each other, thus all the four vertices have to have different colours. Therefore, Rouse Ball and Coxeter's (1987) colouring problem is equivalent to ours, and both have the same solution.

\section{Concluding remarks}

Based on the reasoning in Section 3, and in practice, it is easy to produce the four different solutions to the problem of colouring the hexagons of the truncated icosahedron with five colours. Consider five equal regular tetrahedra, and colour them each differently. Then make their compound, as in Fig. 9. The vertices of the tetrahedra uniquely provide colours to the vertices of the regular dodecahedron. This is the first solution. The second is obtained by interchanging the colours of two tetrahedra. The mirror image of the first and second solutions results in the third and fourth solutions. There is a one-to-one correspondence between the vertices of the dodecahedron and the hexagonal faces of the truncated icosahedron. In this manner, the four different ways of colouring the hexagons of the truncated icosahedron with five colours are determined. The unknown designer of the Wörthsee ball apparently failed to recognize the importance of the compound of five tetrahedra in the colouring problem.

The Wörthsee ball was a coated beach-ball, the design of which required mathematical knowledge, inventive imagination, construction skills, with its realisation needing an ability to work with textiles, and skills in cutting and sewing. The ball was an unnoticed invention, and we do not know where the idea came from, nor who designed and manufactured it. Instead of speculating, there are some undisputed facts.

In the Wörthsee episode of the documentary (Kloft, 2017), among the bathers, there appeared a teenage girl playing with the ball. She was Gertraud Winkler from Jena. She was interested in mathematics, and later studied physics at the University of Jena. Her aunt, who worked as a seamstress before her marriage, was also among the bathers. Gertraud's father was an engineer, who worked in the research and development department of the Zeiss factory in Jena (Knauss, 2002). The scientific member of the board of management of the Zeiss factory was Walter Bauersfeld, who is best known in architecture and structural engineering for designing the first geodesic dome (Fig. 12), which, after concreting, was used as a screen for testing the Zeiss planetarium. His main achievement was the development of the Zeiss planetarium itself, which was the first projector planetarium in the world. The star sphere of the planetarium theoretically was a truncated icosahedron with a projector

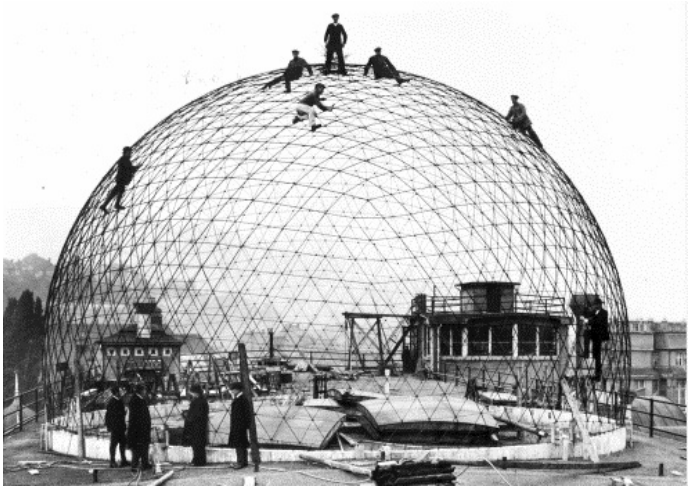

Fig. 12 Steel framework of a 16-m-diameter planetarium test dome, Zeiss Factory Jena, Germany. Design by Walter Bauersfeld and Franz Dischinger, 1922. (Photo: IL Archives Stuttgart, courtesy of Dr. Jürgen Hennike)

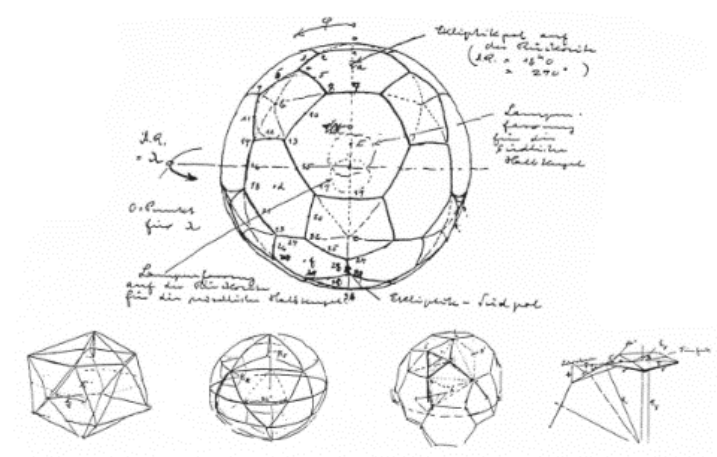

Fig. 13 Bauersfeld's sketch of the projection principle of the star sphere of the planetarium. (Krausse, 1993)

on each face except one where the star sphere was connected to the main body of the planetarium. In an early sketch made by Bauersfeld, the spherical projection of the star sphere appeared as a classical 32-panel soccer ball (Fig. 13). (It should be noted that, in the final model (Fig. 14) set up in Munich in 1924, the star sphere was not Archimedean (Bauersfeld, 1924), but was a truncated icosahedron that had an insphere, that is, all faces had a circumcircle with the same radius (Fig. 15(a), and in this respect, looked like a modern soccer ball where the hexagons are not regular (Fig. 15(b)) (Lengyel and Hincz, 2009).)

\section{Acknowledgement}

We thank Prof. Zsigmond Ritoók and Dr. Endre Makai for much appreciated discussions. We are grateful to Prof. Patrick Fowler for valuable comments and suggestions, to Prof. Zhong You for help with Chinese texts, to Dr. Joseph Clinton for help with Fig. 13 and to Mr. Michael Karlsen for information. Research reported here was supported by the National Research, Development and Innovation Fund (NKFI) grant no. K119440. 


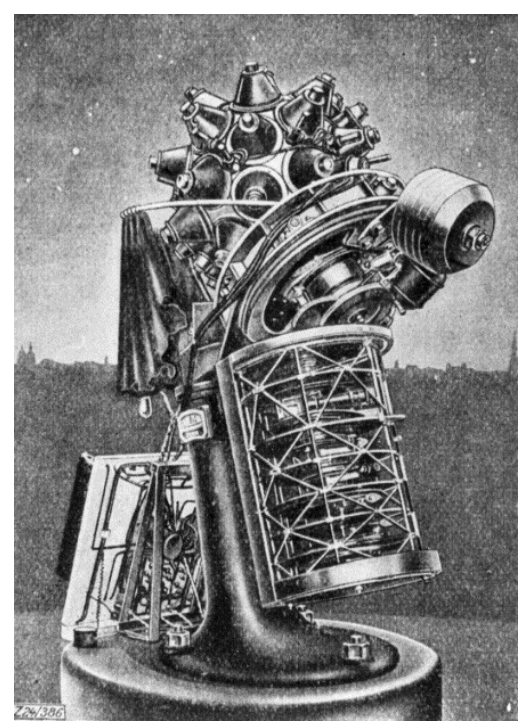

Fig. 14 The Bauersfeld planetarium set up in Munich in 1924. The star sphere is at the top, where the cones are projectors.

(Bauersfeld, 1924)

\section{References}

Bauersfeld, W. (1924) "Das Projektions-Planetarium des Deutschen Museums in München", (The projection-planetarium of the German Museum in Munich) Zeitschrift des Vereines Deutscher Ingenieure, 68(31), pp. 793-797. (in German)

British Museum. (2017) "Toy ball made of linen, hollow, exterior elaborated with red and green segmented decoration formed by strips of reed. Museum number: EA46709, Roman period, found / acquired in Egypt in 1907", [online] Available at: http://www.britishmuseum.org/research/collection_online/search.aspx?searchText=toy/ game-ball [Accessed: 5th September 2018]

Coxeter, H. S. M. (1961) "Introduction to Geometry", 1st ed., Wiley, New York, USA.

Coxeter, H. S. M. (1973) "Regular Polytopes", 3rd ed., Dover Publications, New York, USA.

Cui, L. (1991) "中国古代蹴鞠的起源与发展 《中原文物》1991年 第 2期 | 崔乐泉", (The origin and development of cuju in ancient China) Cultural Relics of Central China, 2, pp. 59-66. (in Chinese) Cundy, H. M., Rollett, A. P. (1981) "Mathematical Models", 3rd ed., Tarquin Publications, Stradbroke, United Kingdom, pp. 82-83.

Doss, M. (1964) "Soccer ball", US Patent, Patent No. Des. 199,535.

Fejes Tóth, L. (1972) "Extremaleigenschaften der regulären Polyeder", (Extreme properties of regular polyhedra) In: Lagerungen in der Ebene auf der Kugel und im Raum, (Packings in the plane, on the sphere and in the space) 2nd ed., Springer-Verlag, Berlin, Heidelberg, Germany, p. 114. (in German)

hwjyw.com "Cuju: a rise and fall of the history of Chinese football", (in Chinese) [online] Available at: http://www.hwjyw.com/zhwh/content/2011/03/24/16171_5.shtml [Accessed: 5th September 2018]

Kloft, M. (2017) "Eva Braun - Die Braut des Bösen: Heimliche Geliebte", (Eva Braun - The bride of evil: secret lovers) Documentary movie, Spiegel TV, zdfinfo, (in German) [online] Available at: https://www.youtube.com/watch?v=hRk_893XsKw [Accessed: 9th April 2018]

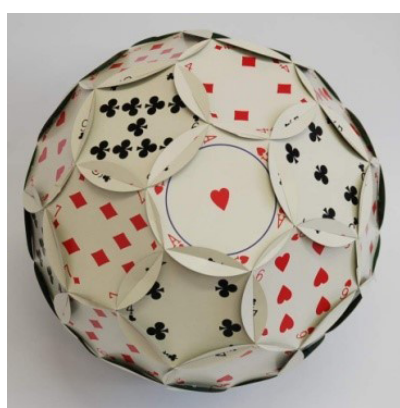

(a)

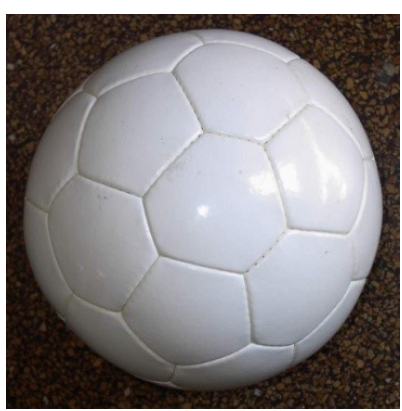

(b)
Fig. 15 Truncation of a regular icosahedron such that the circumcircles of the pentagonal and the hexagonal faces are equal.

(a) A cardboard model. (Photo: Lengyel).

(b) A realisation as a soccer ball. (Photo: Tarnai).

Knauss, S. (2002) "Eva's Cousin", 1st American ed., Ballantine Books, Random House, New York, USA.

Krausse, J. (1993) "Das Wunder von Jena: Das Zeiss-Planetarium von Walter Bauersfeld", (The wonder of Jena: Walter Bauersfeld's Zeiss-Planetarium) $\mathrm{ARCH}^{+}, 116$, pp. 40-49. (in German)

Lengyel, A., Hincz, K. (2009) "Optimization of the roundness of the soccer ball", In: Proceedings of the International Association for Shell and Spatial Structures (IASS) Symposium 2009, Valencia, Spain, pp. 1100-1110.

Newman, J., Pevsner, N. (1975) "The Buildings of England: Dorset", 1st ed., Penguin Books, Harmondsworth, United Kingdom, p. 470.

Pacioli, L. (1509) "Divina proportione", (Divine proportion) A. Paganius Paganinius, Venice, Italy. (in Italian) [online] Available at: https://archive.org/details/divinaproportion00paci [Accessed: 5th September 2018]

Plato (1892a) "Phaedo", In: Jowett, B. (ed.) The Dialogues of Plato, Translated into English with Analyses and Introductions, 2, 3rd ed., Oxford University Press, London, Great Britain, pp. 157266, especially p. 258 (Stephanus number 110b). [online] Available at: http://1f-oll.s3.amazonaws.com/titles/766/0131-02_Bk.pdf [Accessed: 6th September 2018]

Plato (1892b) "Timaeus", In: Jowett, B. (ed.) The Dialogues of Plato, Translated into English with Analyses and Introductions, 3, 3rd ed., Oxford University Press, London, Great Britain, pp. 339-516, especially p. 475 (Stephanus number 55c). [online] Available at: http://1f-oll.s3.amazonaws.com/titles/767/0131-03_Bk.pdf [Accessed: 6th September 2018]

Rassat, A., Thuillier, J.-P. (1996) "A Fullerene-Like Ball in a Second Century Roman Mosaic?", Fullerene Science and Technology, 4(6), pp. 1087-1090. https://doi.org/10.1080/10641229608001167 
Robin, L. (1935) "Chapitre V. Le Monde, l'âme et la divinité", (Chapter V. The world, the soul and divinity) In: Platon, (Plato) Les Échos du Maquis, [pdf] p. 147. (in French) Available at: https://philosophie.cegeptr.qc.ca/wp-content/documents/Platon-1935.pdf [Accessed: 5th September 2018]

Rouse Ball, W. W., Coxeter, H. S. M. (1987) "Chapter VIII. Mapcolouring problems", In: Mathematical Recreations and Essays, 13th ed., Dover Publications, New York, USA, p. 242.

Select-Sport (a) "The Story of SELECT Sport A/S", Available at: http://www.select-sport.com/en/about-select [Accessed: 05 September 2018]

Select-Sport (b) "The Original 32 Panel Ball Construction", Available at: http://www.select-sport.com/en/football-construction [Accessed: 5th September 2018]

EvaPresent WebShop "Strandlabda", (Beach-ball) (in Hungarian) [online] Available at: https://www.evapresent.eu/ [Accessed: 9th April 2018]

Tarnai, T. (2005) "Cutting patterns for inflatables: Soccer ball designs", In: Michailescu, M., Mircea, C. (eds.) IASS 2005 Proceedings of the International Symposium on Shell and Spatial Structures: Theory, Technique, Valuation, Maintenance, Bucharest, Romania, pp. $765-772$.
Tarnai, T., Krähling, J. (2008) "Polyhedra in churches", In: Oliva Salinas, J. G. (ed.) IASS-SLTE 2008 Proceedings of the International Symposium: New Materials and Technologies - New Designs and Innovations, Acapulco, Mexico, CD-ROM

The Stirling Smith Art Gallery and Museum "The World's Oldest Football", [online] Available at: http://www.smithartgalleryandmuseum.co.uk/about/the-collection/stirling-history-archaeology/ the-worlds-oldest-football/ [Accessed: 5th September 2018]

Wenninger, M. J. (1974) "Polyhedron Models", 1st paperback ed., Cambridge University Press, Cambridge, United Kingdom.

Wikipedia (a) "Hosohedron", [online] Available at: https://en.wikipedia. org/wiki/Hosohedron [Accessed: 5th September 2018]

Wikipedia (b) "Villa Romana del Casale, Bikini mosaic", [online] Available at: https://en.wikipedia.org/wiki/Villa_Romana_del_ Casale [Accessed: 5th September 2018]

Wikipedia (c) "Royal Shrovetide Football", [online] Available at: https:// en.wikipedia.org/wiki/Royal_Shrovetide_Football [Accessed: 5th September 2018]

Wikipedia (d) "Adidas", (Adidas) (in German) [online] Available at: https:// de.wikipedia.org/wiki/Adidas [Accessed: 5th September 2018]

Wikipedia (e) "Permutation", [online] Available at: https://en.wikipedia. org/wiki/Permutation [Accessed: 5th September 2018] 